\title{
Canonical orbits for rapidly deforming planar microswimmers in shear flow
}

\author{
Eamonn A. Gaffney,,$^{1, *}$ Mohit P. Dalwadi, ${ }^{1,2, \dagger}$ Clément Moreau $\odot,{ }^{3, 末}$ \\ Kenta Ishimoto, ${ }^{3, \S}$ and Benjamin J. Walker ${ }^{1,2, \|}$ \\ ${ }^{1}$ Mathematical Institute, University of Oxford, Oxford, OX2 6GG, United Kingdom \\ ${ }^{2}$ Department of Mathematics, University College London, London, WC1H OAY, United Kingdom \\ ${ }^{3}$ Research Institute for Mathematical Sciences, Kyoto University, Kyoto, 606-8502, Japan
}

(Received 2 November 2021; accepted 21 January 2022; published 18 February 2022)

\begin{abstract}
Classically, the rotation of ellipsoids in shear Stokes flow is captured by Jeffery's orbits. Here we demonstrate that Jeffery's orbits also describe high-frequency shape-deforming swimmers moving in the plane of a shear flow, employing only basic properties of Stokes flow and a multiple-scales asymptotic analysis. In doing so, we support the use of these simple models for capturing shape-changing swimmer dynamics in studies of active matter and highlight the ubiquity of ellipsoid-like dynamics in complex systems. This result is robust to weakly confounding effects, such as distant boundaries, and also applies in the low-frequency limit.
\end{abstract}

DOI: 10.1103/PhysRevFluids.7.L022101

In classical mechanics, it is well known that the rotational dynamics of rigid bodies can be reduced to those of ellipsoids via the moment of inertia tensor. A similar correspondence holds between rigid bodies and ellipsoids in Stokesian fluid dynamics, with the angular motion of a broad class of particles in shear flow known to be equivalent simply to those of an ellipsoid. In this zero-Reynolds-number setting, it is the celebrated work of Jeffery [1] that establishes analytical solutions for the rotation of ellipsoids in shear flow, which has been explicitly linked to the motion of a wide range of rigid bodies in subsequent generalizations [2-5]. However, looking beyond rigid particles, it is unclear how far the scope of the so-called Jeffery's orbit solution extends. Recently, numerical studies have suggested that such a description may even apply to complex shape-deforming microswimmers, including the well-studied spermatozoon [6,7]. These small-scale swimmers are characterised by low-Reynolds-number mechanics and rapidly evolving geometries, with the deformation of a slender flagellum canonically driving the motion of spermatozoa, for instance. Analogous relevant examples also include motile kinetoplastid pathogens, such as Trypanosoma and Leishmania, as well as Volvox and artificial shape-deforming swimmers. Owing to the changing morphology of such swimmers, the methodology used to establish links between ellipsoids and rigid bodies cannot simply be extended to include shape-deforming

\footnotetext{
*gaffney@maths.ox.ac.uk

†m.dalwadi@ucl.ac.uk

${ }^{+}$cmoreau@kurims.kyoto-u.ac.jp

§ishimoto@kurims.kyoto-u.ac.jp

"Corresponding author: benjamin.walker@ucl.ac.uk
}

Published by the American Physical Society under the terms of the Creative Commons Attribution 4.0 International license. Further distribution of this work must maintain attribution to the author(s) and the published article's title, journal citation, and DOI. 


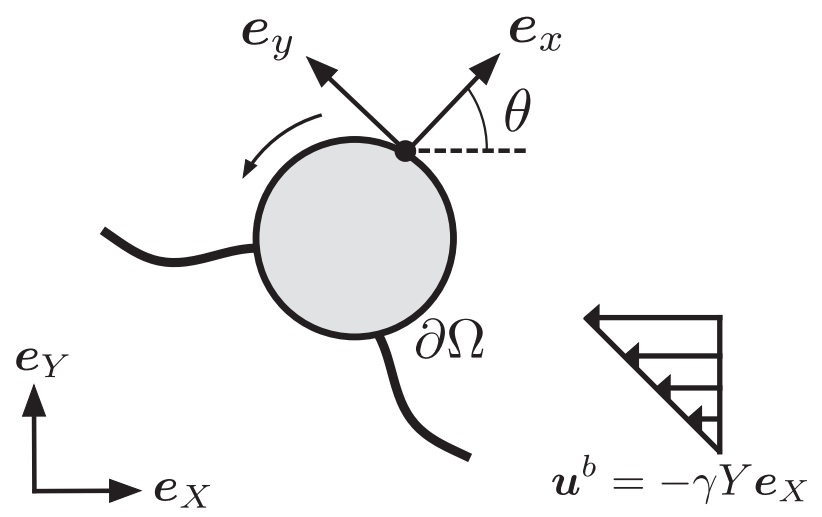

FIG. 1. Swimmer and laboratory frames. A generic swimmer with time-dependent boundary $\partial \Omega$ is shown along with the basis $\left\{\boldsymbol{e}_{x}, \boldsymbol{e}_{y}, \boldsymbol{e}_{z}\right\}$ of a swimmer-fixed frame. The swimmer frame is instantaneously at an angle $\theta$ to the laboratory frame $\left\{\boldsymbol{e}_{X}, \boldsymbol{e}_{Y}, \boldsymbol{e}_{Z}\right\}$, which corresponds to rotation about the shared $\boldsymbol{e}_{z}=\boldsymbol{e}_{Z}$ axis in the $\boldsymbol{e}_{X} \boldsymbol{e}_{Y}$ plane of swimmer motion.

swimmers; indeed, it is not even clear that a correspondence exists beyond select numerical examples.

In addition to complementing numerical observations, establishing such a link has the potential to further our fundamental understanding into the complex and well-studied dynamics of commonplace swimmers in background flows, which have been the subject of significant classical and recent enquiry [6,8-12]. Further, in the wide context of the study of active biological matter, this connection would afford rigorous theoretical justification to past and future uses of simple models in approximating shape-changing swimmers in flow [7,13-19], potentially enabling analytical progress in settings previously limited to numerical exploration.

In this Letter, we will seek to realize the above and establish the existence of a connection between Jeffery's orbits and the angular dynamics of rapidly deforming swimmers in the plane of a shear flow. Our approach will employ classical results of Stokesian fluid mechanics coupled to a multiple-scales asymptotic analysis, bypassing the details of the complex hydrodynamic problem associated with shape-changing swimmers. Our key result, which we later extend, is captured in the following proposition:

Proposition. Consider a lone drift-free swimmer at zero Reynolds number that moves in the plane of a shear flow with shear rate $\gamma$, without roll or pitch, whose shape periodically evolves with frequency $\omega \gg \gamma$. Then, if there is no motion out of the plane of the shear flow, the average rotational motion of the swimmer in an unbounded domain is given by a Jeffery's orbit with relative asymptotic error of $O(\gamma / \omega)$.

Proceeding more precisely, consider an isolated swimmer with boundary $\partial \Omega$ whose gait evolves on the fast timescale $T=\omega t$ with period $2 \pi$, where $\omega \gg 1$ and all quantities are dimensionless here and hereafter. We assume that, in the absence of external flow, the gait of the swimmer contributes no net change to its orientation over a period, a condition that we will later formulate explicitly. We define a background shear flow $\boldsymbol{u}^{b}=-\gamma Y \boldsymbol{e}_{X}$ in the fluid domain $\Omega$ that is parallel to the $\boldsymbol{e}_{X} \boldsymbol{e}_{Y}$ plane of an inertial laboratory frame, with $\left\{\boldsymbol{e}_{X}, \boldsymbol{e}_{Y}, \boldsymbol{e}_{Z}\right\}$ being a right-handed orthonormal basis and where $\gamma$ is the dimensionless shear rate, assumed to be of order unity. Separately, we define a swimmer-fixed frame with origin $X_{O}(T, t) \boldsymbol{e}_{X}+Y_{O}(T, t) \boldsymbol{e}_{Y}$ in the laboratory frame and an associated swimmer-fixed orthonormal basis $\left\{\boldsymbol{e}_{x}, \boldsymbol{e}_{y}, \boldsymbol{e}_{z}\right\}$, where $\boldsymbol{e}_{z} \equiv \boldsymbol{e}_{Z}$ and $\boldsymbol{e}_{x}=\cos \theta \boldsymbol{e}_{X}+\sin \theta \boldsymbol{e}_{Y}$ is at an angle $\theta(T, t)$ from $\boldsymbol{e}_{X}$, as illustrated in Fig. 1. To facilitate a later multiple-scales asymptotic analysis, we distinguish between functions of the fast gait timescale, $T$, and functions of the slow flow timescale, $t$, writing these dependencies explicitly and distinctly throughout. Notably, the surface $\partial \Omega$ of the swimmer is a function only of the fast timescale $T$ when viewed in the swimmer-fixed 
frame. In particular, a point $\boldsymbol{p} \in \partial \Omega$ has position

$$
\boldsymbol{x}^{S}(\boldsymbol{p}, T)=x^{S}(\boldsymbol{p}, T) \boldsymbol{e}_{x}+y^{S}(\boldsymbol{p}, T) \boldsymbol{e}_{y}+z^{S}(\boldsymbol{p}) \boldsymbol{e}_{z}
$$

and velocity

$$
\boldsymbol{U}^{S}(\boldsymbol{p}, T)=\omega \boldsymbol{x}_{T}^{S}=\omega\left(x_{T}^{S} \boldsymbol{e}_{x}+y_{T}^{S} \boldsymbol{e}_{y}\right),
$$

both relative to the swimmer frame, using superscripts of $S$ to denote quantities relating to the swimmer surface. Here, subscripts of $T$ denote partial differentiation with respect to $T$ and we note that $z_{T}^{S}$ is zero, given no motion out of the plane of the shear flow. The velocity of the shear flow in the laboratory frame $\boldsymbol{u}^{b}(\boldsymbol{p}, T, t)$ at a surface point $\boldsymbol{p}$ is then simply

$$
\boldsymbol{u}^{b}(\boldsymbol{p}, T, t)=-\gamma(Y_{O}(T, t)+\underbrace{x^{S}(\boldsymbol{p}, T) \sin \theta+y^{S}(\boldsymbol{p}, T) \cos \theta}_{\boldsymbol{e}_{Y} \cdot \boldsymbol{x}^{S}(\boldsymbol{p}, T)}) \boldsymbol{e}_{X} .
$$

The relative fluid velocity at $\boldsymbol{p}$, denoted $\boldsymbol{U}^{r}(\boldsymbol{p}, T, t)$, is then given by the difference between the swimmer velocity in the laboratory frame and the background shear velocity. Defining $\tilde{\boldsymbol{x}}^{S}=\boldsymbol{e}_{z} \wedge$ $\boldsymbol{x}^{S}=-y^{S} \boldsymbol{e}_{x}+x^{S} \boldsymbol{e}_{y}$ and expressing the velocity of the origin of the swimmer frame relative to the laboratory frame as $u(T, t) \boldsymbol{e}_{x}+v(T, t) \boldsymbol{e}_{y}$ in the swimmer basis [20], the relative velocity at $\boldsymbol{p}$ is

$$
\begin{aligned}
\boldsymbol{U}^{r}(\boldsymbol{p}, T, t) & =u \boldsymbol{e}_{x}+v \boldsymbol{e}_{y}+\dot{\theta} \boldsymbol{e}_{z} \wedge \boldsymbol{x}^{S}+\boldsymbol{U}^{S}-\boldsymbol{u}^{b} \\
& =u \boldsymbol{e}_{x}+v \boldsymbol{e}_{y}+\dot{\theta} \tilde{\boldsymbol{x}}^{S}+\omega \boldsymbol{x}_{T}^{S}+\gamma\left(Y_{O}+x^{S} \sin \theta+y^{S} \cos \theta\right) \boldsymbol{e}_{X},
\end{aligned}
$$

where an overdot denotes a full derivative with respect to time, which we will later consider in the context of a multiple scales analysis, and we have omitted arguments for brevity. As imposed, we note that $\boldsymbol{e}_{z} \cdot \boldsymbol{U}^{r}=0$.

Applying the no-slip condition on the surface of the swimmer, $\boldsymbol{U}^{r}$ becomes the surface velocity of a Stokes problem in the domain $\Omega$. Therefore, there exists a linear operator $\mathcal{L}(T)$ such that $\boldsymbol{f}=\mathcal{L}(T) \boldsymbol{U}^{r}$, where $\boldsymbol{f}=\boldsymbol{f}(\boldsymbol{p}, T, t)$ is the instantaneous traction field on the boundary $\partial \Omega$ [21]. Subject to the removal of the pressure gauge freedom inherent in Stokes flows, which will have no effect on this swimming problem, the traction is uniquely determined by the surface velocity and the linear operator is, therefore, well defined. By the translational and rotational independence of the Stokes equations, this operator is invariant to both translation and rotation, depending only on the geometry of the domain and, therefore, only on the geometry of the swimmer, recalling that our swimmer is moving in free space. In particular, if both $\boldsymbol{f}$ and $\boldsymbol{U}^{r}$ are expressed in the swimmer basis, then the action of $\mathcal{L}(T)$ is readily seen to be independent of $\theta$ in this free-space swimming problem, recalling that $\partial \Omega=\partial \Omega(T)$ in the swimmer-fixed frame. For brevity, we will suppress the explicit dependence of the operator on $T$ hereafter, writing $\mathcal{L}(T)=\mathcal{L}$.

Next, we impose the force- and torque-free conditions appropriate to low-Reynolds-number swimming, which we can succinctly write as

$$
\begin{aligned}
& \mathbf{0}=\langle\boldsymbol{f}\rangle=\left\langle\mathcal{L} \boldsymbol{U}^{r}\right\rangle, \\
& \mathbf{0}=\left\langle\boldsymbol{x}^{S} \wedge \boldsymbol{f}\right\rangle=\left\langle\boldsymbol{x}^{S} \wedge \mathcal{L} \boldsymbol{U}^{r}\right\rangle,
\end{aligned}
$$

taking moments about the origin of the swimmer frame and having defined

$$
\langle q\rangle:=\iint_{\partial \Omega} q d S_{\partial \Omega}
$$

to be the integral of the quantity $q$ over $\partial \Omega$. Note that we are free to resolve moments about the origin of the swimmer frame owing to the force-free condition on the swimmer. Though the above seemingly constitute six scalar equations, we note that the $\boldsymbol{e}_{z}$ component of the force balance and the $\boldsymbol{e}_{x}$ and $\boldsymbol{e}_{y}$ components of the moment balance will automatically be satisfied due to the assumed planarity of the swimming motion. Thus, we may safely neglect these equations and, 
correspondingly, the $\boldsymbol{e}_{z}$ components of the traction $\boldsymbol{f}$, defining the projected operator $\hat{\mathcal{L}}$ to be the result of applying $\mathcal{L}$ then projecting onto the $\boldsymbol{e}_{x} \boldsymbol{e}_{y}$ plane, which we note inherits linearity and both rotational and translational invariance within the shear plane from the properties of $\mathcal{L}$. With this projected operator, we have the reduced force- and torque-balance equations

$$
\begin{aligned}
& \mathbf{0}=\left\langle\hat{\mathcal{L}} \boldsymbol{U}^{r}\right\rangle, \\
& 0=\boldsymbol{e}_{z} \cdot\left\langle\boldsymbol{x}^{S} \wedge \hat{\mathcal{L}} \boldsymbol{U}^{r}\right\rangle,
\end{aligned}
$$

a system of three scalar equations. Before inserting our expression for $\boldsymbol{U}^{r}$ into these conditions, it is notationally convenient to define three matrices of vector-valued functions

$$
\begin{aligned}
\mathcal{M} & =\left(\hat{\mathcal{L}} \boldsymbol{e}_{x} \mid \hat{\mathcal{L}} \boldsymbol{e}_{y}\right), \\
\mathcal{W}_{x} & =\left(\begin{array}{l|l}
\hat{\mathcal{L}} x^{S} \boldsymbol{e}_{x} \mid \hat{\mathcal{L}} x^{S} \boldsymbol{e}_{y}
\end{array},\right. \\
\mathcal{W}_{y} & =\left(\hat{\mathcal{L}} y^{S} \boldsymbol{e}_{x} \mid \hat{\mathcal{L}} y^{S} \boldsymbol{e}_{y}\right),
\end{aligned}
$$

each a function of $\boldsymbol{p}$ and $T$ and where a bar separates column vectors. For clarity, we remark that $\hat{\mathcal{L}} x^{S} \boldsymbol{e}_{x}$ denotes the application of the $T$-dependent operator $\hat{\mathcal{L}}$ to the field $x^{S} \boldsymbol{e}_{x}$, with other such terms being interpreted analogously. The condition of force-free swimming can then be expanded as

$$
\mathbf{0}=\left\langle\left(\mathcal{M} \mid \hat{\mathcal{L}} \tilde{\boldsymbol{x}}^{S}\right)\right\rangle\left(\begin{array}{c}
u \\
v \\
\dot{\theta}
\end{array}\right)+\omega\left\langle\hat{\mathcal{L}} \boldsymbol{x}_{T}^{S}\right\rangle+\gamma\left[Y_{O}\langle\mathcal{M}\rangle+\sin \theta\left\langle\mathcal{W}_{x}\right\rangle+\cos \theta\left\langle\mathcal{W}_{y}\right\rangle\right]\left(\begin{array}{c}
\cos \theta \\
-\sin \theta
\end{array}\right),
$$

exploiting the linearity of both $\hat{\mathcal{L}}$ and the spatial integral operator $\langle\cdot\rangle$.

The scalar moment balance equation can be written as

$$
0=\boldsymbol{e}_{z} \cdot\left\langle\boldsymbol{x}^{S} \wedge \mathcal{L} \boldsymbol{U}^{r}\right\rangle=\left\langle\tilde{\boldsymbol{x}}^{S} \cdot \hat{\mathcal{L}} \boldsymbol{U}^{r}\right\rangle
$$

by the circular property of the scalar triple product [22]. Noting the similarity of this last expression to the force balance equation, we once again exploit the linearity of the integral and hydrodynamic operators and combine the expanded expressions for the force- and torque-free equations into a single system:

$$
\begin{aligned}
\mathbf{0}= & \underbrace{\left\langle\left(\begin{array}{c}
\mathcal{M} \\
\tilde{\boldsymbol{x}}^{S} \cdot \mathcal{M}
\end{array} \begin{array}{c}
\hat{\mathcal{L}} \tilde{\boldsymbol{x}}^{S} \\
\tilde{\boldsymbol{x}}^{S} \cdot \hat{\mathcal{L}} \tilde{\boldsymbol{x}}^{S}
\end{array}\right)\right\rangle}_{\boldsymbol{A}(T)}\left(\begin{array}{c}
u \\
v \\
\dot{\theta}
\end{array}\right)+\omega\left\langle\left(\begin{array}{c}
\hat{\mathcal{L}} \boldsymbol{x}_{T}^{S} \\
\tilde{\boldsymbol{x}}^{S} \cdot \hat{\mathcal{L}} \boldsymbol{x}_{T}^{S}
\end{array}\right)\right\rangle \\
& +\gamma\left[Y_{O}\left\langle\left(\begin{array}{c}
\mathcal{M} \\
\tilde{\boldsymbol{x}}^{S} \cdot \mathcal{M}
\end{array}\right)\right\rangle+\sin \theta\left\langle\left(\begin{array}{c}
\mathcal{W}_{x} \\
\tilde{\boldsymbol{x}}^{S} \cdot \mathcal{W}_{x}
\end{array}\right)\right\rangle+\cos \theta\left\langle\left(\begin{array}{c}
\mathcal{W}_{y} \\
\tilde{\boldsymbol{x}}^{S} \cdot \mathcal{W}_{y}
\end{array}\right)\right\rangle\right]\left(\begin{array}{c}
\cos \theta \\
-\sin \theta
\end{array}\right) .
\end{aligned}
$$

Here and throughout, we are defining $\boldsymbol{a} \cdot \boldsymbol{b}:=\boldsymbol{a}^{\dagger} \boldsymbol{b}$, where $\boldsymbol{a}^{\dagger}$ is the transpose of the vector $\boldsymbol{a}$ and $\boldsymbol{b}$ is a vector or a matrix, a natural generalization of the usual dot product of vectors. The first term of Eq. (2) represents the forces and torques generated by rigid body motion, while the second and third terms encode the effects of the deformation and the background flow, respectively. Defining $A$ to be the $T$-dependent matrix operator that acts on the swimmer velocities, we note that $\boldsymbol{A}$ acts as a resistance matrix and is invertible by standard energy conservation arguments [23]. Writing the entries of $\boldsymbol{A}$ with respect to the swimmer basis, we also note that $\boldsymbol{A}$ is independent of the swimmer orientation $\theta$, a property that it inherits from $\mathcal{L}$. Hence, $\boldsymbol{A}$ also depends only on the swimming gait and is, therefore, a function purely of $T$.

Seeking a solution for $\dot{\theta}$, we apply the inverse operator $\boldsymbol{A}^{-1}$ to Eq. (2) and define $\boldsymbol{\alpha}=(0,0,1) \boldsymbol{A}^{-1}$ to concisely take the third component of the resulting vector equation, giving

$$
\dot{\theta}=-\omega \boldsymbol{\alpha}\left\langle\left(\begin{array}{c}
\hat{\mathcal{L}} \boldsymbol{x}_{T}^{S} \\
\tilde{\boldsymbol{x}}^{S} \cdot \hat{\mathcal{L}} \boldsymbol{x}_{T}^{S}
\end{array}\right)\right\rangle-\gamma \boldsymbol{\alpha}\left[\sin \theta\left\langle\left(\begin{array}{c}
\mathcal{W}_{x} \\
\tilde{\boldsymbol{x}}^{S} \cdot \mathcal{W}_{x}
\end{array}\right)\right\rangle+\cos \theta\left\langle\left(\begin{array}{c}
\mathcal{W}_{y} \\
\tilde{\boldsymbol{x}}^{S} \cdot \mathcal{W}_{y}
\end{array}\right)\right\rangle\right]\left(\begin{array}{c}
\cos \theta \\
-\sin \theta
\end{array}\right)
$$


Notably, the $Y_{O}$ term has been annihilated by the premultiplication by $\boldsymbol{\alpha}$. This follows from the equality between the first two columns of $\boldsymbol{A}(T)$ and the matrix coefficient of $Y_{O}$ in Eq. (2), noting that, for a general $3 \times 3$ invertible matrix with columns $\boldsymbol{a}, \boldsymbol{b}$, and $\boldsymbol{c}$, we have

$$
(\boldsymbol{a}|\boldsymbol{b}| \boldsymbol{c})^{-1}(\boldsymbol{a} \mid \boldsymbol{b})=\left(\begin{array}{cc}
1 & 0 \\
0 & 1 \\
0 & 0
\end{array}\right)
$$

by the basic requirements of the inverse operator. Hence, the angular dynamics decouple from the location of the swimmer frame relative to the origin of the shear flow.

Significantly, since $x^{S}, y^{S}$, and $\hat{\mathcal{L}}$ depend only on the fast timescale $T$, Eq. (3) for $\dot{\theta}$ depends on $t$ only through $\theta$. Further, this $\theta$ dependence only arises through the terms $\sin \theta \cos \theta, \sin ^{2} \theta$, and $\cos ^{2} \theta$. Hence, by the double-angle formulas, we may write the governing equation for $\dot{\theta}$ in the succinct form

$$
\dot{\theta}=\gamma[\xi(T)-\eta(T) \cos (2 \theta)-\chi(T) \sin (2 \theta)]+\omega g(T),
$$

explicitly emphasizing that the term involving $\omega$ does not depend on $\theta$ or $\gamma$.

We now pursue a multiple-scales analysis [24], exploiting the assumed separation between the fast timescale $T=\omega t$ and the timescale $t$ of the shear-driven dynamics, with $\omega \gg 1$ and $\gamma=O(1)$. Accordingly, the temporal derivative operator transforms as

$$
\frac{d}{d t}=\frac{\partial}{\partial t}+\omega \frac{\partial}{\partial T}
$$

and we expand $\theta$ in inverse powers of $\omega$ as

$$
\theta=\theta_{0}(T, t)+\frac{1}{\omega} \theta_{1}(T, t)+O\left(\frac{1}{\omega^{2}}\right) .
$$

We also define

$$
\bar{a}=\frac{1}{2 \pi} \int_{0}^{2 \pi} a(T) d T
$$

as the fast-timescale average of the quantity $a$, which we will make use of below, recalling that the swimmer gait is assumed to be periodic with period $2 \pi$.

Using the asymptotic expansion of Eq. (5) in Eq. (4) and equating coefficients of $\omega$, the leadingorder angular dynamics are simply

$$
\frac{\partial \theta_{0}}{\partial T}=g(T) \Rightarrow \theta_{0}(T, t)=\bar{\theta}_{0}(t)+G(T),
$$

where $G(T)$ is the antiderivative of $g(T)$. Our assumption of drift-free angular dynamics in the absence of flow translates directly into $\bar{g}=0$, which leads to $G(T)$ being periodic. Such dynamics are known to be exhibited by all swimmers with antiperiodic swimming gaits, as defined and discussed by Walker et al. [7, Appendix C], for example. With this assumption, we are free to choose $G(T)$ to have zero fast-timescale average without loss of generality, so that the $t$ dependence of $\theta_{0}$ is precisely that of its temporal average $\bar{\theta}_{0}(t)$. At next order, we have

$$
\begin{aligned}
\frac{\partial \theta_{0}}{\partial t}+\frac{\partial \theta_{1}}{\partial T} & =\gamma\left[\xi(T)-\eta(T) \cos \left(2 \theta_{0}\right)-\chi(T) \sin \left(2 \theta_{0}\right)\right] \\
& =\gamma\{\xi-\underbrace{[\eta \cos (2 G)+\chi \sin (2 G)]}_{\alpha(T)} \cos \left(2 \bar{\theta}_{0}\right)-\underbrace{[\chi \cos (2 G)-\eta \sin (2 G)]}_{\beta(T)} \sin \left(2 \bar{\theta}_{0}\right)\},
\end{aligned}
$$

having suppressed the $T$ dependence of $\xi, \eta, \chi$, and $G$ in the last expression for brevity. Imposing that $\theta_{1}$ is $T$-periodic, as is standard in the method of multiple scales [24], we average over a period 
in $T$ to obtain

$$
\frac{d \bar{\theta}_{0}}{d t}=\gamma\left[\bar{\xi}-\bar{\alpha} \cos \left(2 \bar{\theta}_{0}\right)-\bar{\beta} \sin \left(2 \bar{\theta}_{0}\right)\right],
$$

where $\bar{\xi}, \bar{\alpha}$, and $\bar{\beta}$ are constants and we note the inherited periodicity of $\xi, \eta, \chi$, and $G$ in $T$. For $\bar{\xi} \neq 0$, with $\gamma_{e}:=2 \gamma \bar{\xi}$ as the effective shear rate, this can be written as

$$
\frac{d \bar{\theta}_{0}}{d t}=\frac{\gamma_{e}}{2}\left[1-B \cos \left(2 \bar{\theta}_{0}+2 \theta_{c}\right)\right]
$$

This simple ordinary differential equation is recognizable as that corresponding to a generalized Jeffery's orbit $[2-5,7]$. Hence, excluding the mathematically precise degenerate cases where $\gamma_{e} B=$ 0 , the average angular motion of the deforming swimmer is given by a Jeffery's orbit at leading order in $1 / \omega$, thereby demonstrating the proposition. This orbit is characterized by an effective shear rate $\gamma_{e}$, a phase shift $\theta_{c}$, and a shape-capturing Bretherton constant $B$ [2]. In particular, if $|B|<1$, we can identify this orbit with that of a simple spheroid in the effective shear flow.

A simplified constructive example. As our result above is largely nonconstructive, with explicit expressions being prohibited by the generality of the operator $\mathcal{L}$, its primary utility is in establishing the broad validity of Jeffery's orbit models for deforming swimmers in shear flow. However, subject to simplifying assumptions and approximations, we can make explicit progress in a canonical example. In particular, if our swimmer is a slender filament undergoing small-amplitude deformations away from a straight configuration, we may make use of the classical resistive force theory approximation of Gray and Hancock [25], acknowledging the associated error. In this case, the operator $\mathcal{L}$ is significantly simplified to a purely local matrix operator $\mathbf{L}=-\left(2 \mathbf{I}-\boldsymbol{e}_{x} \otimes \boldsymbol{e}_{x}\right)$, where $\mathbf{I}$ is the identity matrix and the dimensionless tangential resistive coefficient is unity, so that

$$
\boldsymbol{f}(\boldsymbol{p}, T, t) \approx \mathbf{L} \boldsymbol{U}^{r}(\boldsymbol{p}, T, t) .
$$

redefining $f$ here to be the force per unit length on the filament, rather than the surface traction. With the slender-body approximation also entailing that we need only consider the swimmer centerline, we write $\boldsymbol{x}^{S}=-p \boldsymbol{e}_{x}+\delta h(p, T) \boldsymbol{e}_{y}$, with dimensionless arclength parameter $p \in[0,2 \pi]$, gait amplitude $\delta \ll 1$, and gait function $h$ of order unity. A simple but notationally cumbersome computation, given in the Supplemental Material [26] along with an extension to include an attached sphere, yields the angular evolution equation

$$
\dot{\theta}=\frac{\gamma}{2}[1-\cos (2 \theta)]+\frac{3}{2 \pi^{3}} \omega \delta \int_{0}^{2 \pi}(p-\pi) h_{T}(p, T) d p,
$$

where the integral term corresponds to $\omega g(T)$ in Eq. (4), with asymptotic corrections of $O(\gamma \delta)$, $O\left(\omega \delta^{2}\right)$. Equations of this form are investigated in the detailed multiple-scales analysis of Walker et al. [7], wherein it is indeed found that the leading-order average dynamics follow a Jeffery's orbit if $\omega \delta \gg 1$, in this case with unmodified shear rate $\gamma$ and Bretherton constant $B$ of magnitude less than unity, along with a potential shift in definition of swimmer orientation.

Slowly drifting swimmers. In performing the multiple-scales analysis, we assumed that the swimming gait introduces no net change in swimmer orientation over each period, which allowed us to conclude that $\bar{g}=0$. While this assumption holds in many swimming scenarios, some deforming microswimmers can exhibit a relatively small angular drift over a period. For instance, the planar, asymmetric beating of the bovine spermatozoa documented by Friedrich et al. rotates these swimmers by less than $9^{\circ}$ each beat cycle even when tightly circling [27, Fig. 3], alongside analogous qualitative reports from Woolley for a variety of species [28]. We investigate the effect of such a small angular drift over a period in the Supplemental Material [26]. Once again, we recover the angular evolution equation of generalized Jeffery's orbits, highlighting the persistence of Jeffery's orbits across planar swimmers and swimming gaits.

Beyond free-space swimming. In reducing the general angular evolution equation to a form readily amenable to asymptotic analysis, we have exploited the independence of the matrix $\boldsymbol{A}(T)$ 
from the position and rotation of the swimmer in the shear plane, an assumption inherited from $\mathcal{L}$ that is valid in the case of swimming in free space in the plane of a shear flow. However, with swimmers rarely isolated, often moving in the presence of boundaries or even other swimmers, it is pertinent to consider the case where $\mathcal{L}$ might depend on other quantities, so that generically $\mathcal{L}=\mathcal{L}\left(T, t, X_{O}, Y_{O}, \theta, \ldots\right)$. While it is clear that a generalized form of our conclusion will not hold in all contexts, with boundary effects being of obvious importance for a swimmer very close to a no-slip surface, for instance, we can recover a qualified generalization. In particular, noting that the largest terms in the previous analysis are of $O(\omega)$, and that the previous analysis required proceeding to $O(1)$, our free-space result remains valid if we can write $\mathcal{L}=\mathcal{L}_{0}(T)+o(1 / \omega)$, where any additional dependencies of $\mathcal{L}$ are contained within the $o(1 / \omega)$ terms. This condition is equivalent to the notion that the leading-order hydrodynamic problem depends only on $T$, with confounding factors such as the presence of boundaries and other swimmers contributing corrections of magnitude less than $1 / \omega$.

Reduction to slowly deforming or rigid bodies. Though we have focused on the motion of rapidly deforming swimmers, Eq. (4) can be further analysed in two additional cases: slow swimmer evolution, where $\omega \ll 1$, and a rigid body, where $\omega=0$ and $T$ is simply constant. In the case of a rigid body, Eq. (4) readily reduces to the form of a Jeffery's orbit, noting that there is no gait and that $\xi, \eta$, and $\chi$ are constants in this setting. Hence, as a corollary, we have also demonstrated that arbitrary rigid bodies undergo Jeffery's orbits, subject to the condition that they are symmetric in the plane of the background shear flow. The case of slow swimmer evolution, where the body deforms with frequency $\omega \ll 1$, is a simple regular perturbation of the $\omega=0$ case, and therefore gives rise to a Jeffery's orbit solution at $O(1)$, with a natural long-time drift at $O(\omega)$. Hence, the angular dynamics of a slowly deforming body that moves only in the plane of a shear flow are also captured by a Jeffery's orbit at leading order.

Summary and conclusions. Via simple properties of Stokesian hydrodynamics and a multiplescales analysis, we have shown that the dynamics of planar drift-free rapidly deforming swimmers in shear flow are, on average and to leading order, given simply by the Jeffery's orbits of rigid particles. This result is precisely in line with previous numerical observations [6,7], and the assumed planarity of motion and high-frequency gait are both common to many well-studied microswimmers, including some spermatozoa. We have further noted a robustness of this free-space analysis to more-complex hydrodynamic environments and to slowly deforming or rigid bodies, with the presented overall conclusion thereby justifying the judicious use of Jeffery's orbit models for planar swimming in shear flow.

Acknowledgments. The authors are grateful to Prof. Eric Lauga for interesting and motivating discussions on the separated timescales of motion found in many microswimming problems. C.M. was partially supported by the JSPS Postdoctoral Fellowship program (No. PE20021). K.I. acknowledges JSPS-KAKENHI for Young Researchers (Grant No. 18K13456), JSPS-KAKENHI for Transformative Research Areas (Grant No. 21H05309), and JST, PRESTO, Japan (Grant No. JPMJPR1921). C.M. and K.I. were partially supported by the Research Institute for Mathematical Sciences, an International Joint Usage/Research Center located at Kyoto University. B.J.W. is supported by the Royal Commission for the Exhibition of 1851 and the U.K. Engineering and Physical Sciences Research Council (EPSRC), Grant No. EP/R513295/1.

[1] G. B. Jeffery, The motion of ellipsoidal particles immersed in a viscous fluid, Proc. R. Soc. London A 102, 161 (1922).

[2] F. P. Bretherton, The motion of rigid particles in a shear flow at low Reynolds number, J. Fluid Mech. 14, 284 (1962).

[3] H. Brenner, The Stokes resistance of an arbitrary particle-III: Shear fields, Chem. Eng. Sci. 19, 631 (1964). 
[4] K. Ishimoto, Helicoidal particles and swimmers in a flow at low Reynolds number, J. Fluid Mech. 892, A11 (2020).

[5] K. Ishimoto, Jeffery orbits for an object with discrete rotational symmetry, Phys. Fluids 32, 081904 (2020).

[6] B. J. Walker, K. Ishimoto, R. J. Wheeler, and E. A. Gaffney, Response of monoflagellate pullers to a shearing flow: A simulation study of microswimmer guidance, Phys. Rev. E 98, 063111 (2018).

[7] B. J. Walker, K. Ishimoto, E. A. Gaffney, C. Moreau, and M. P. Dalwadi, Effects of rapid yawing on simple swimmer models and planar Jeffery's orbits, Phys. Rev. Fluids 7, 023101 (2022).

[8] F. P. Bretherton and N. M. V. Rothschild, Rheotaxis of spermatozoa, Proc. R. Soc. London B 153, 490 (1961).

[9] V. Kantsler, J. Dunkel, M. Blayney, and R. E. Goldstein, Rheotaxis facilitates upstream navigation of mammalian sperm cells, eLife 3, e03521 (2014).

[10] A. Zöttl and H. Stark, Nonlinear dynamics of a microswimmer in Poiseuille flow, Phys. Rev. Lett. 108, 218104 (2012).

[11] H. Matsui, T. Omori, and T. Ishikawa, Rheology of a dilute suspension of deformable microswimmers, Phys. Fluids 32, 071902 (2020).

[12] A. Hope, O. A. Croze, W. C. K. Poon, M. A. Bees, and M. D. Haw, Resonant alignment of microswimmer trajectories in oscillatory shear flows, Phys. Rev. Fluids 1, 051201(R) (2016).

[13] G. Rosser, R. E. Baker, J. P. Armitage, and A. G. Fletcher, Modelling and analysis of bacterial tracks suggest an active reorientation mechanism in Rhodobacter sphaeroides, J. R. Soc., Interface 11, 20140320 (2014).

[14] D. M. Richards and R. G. Endres, Target shape dependence in a simple model of receptor-mediated endocytosis and phagocytosis, Proc. Natl. Acad. Sci. USA 113, 6113 (2016).

[15] C. Moreau, K. Ishimoto, E. A. Gaffney, and B. J. Walker, Control and controllability of microswimmers by a shearing flow, R. Soc. Open Sci. 8, 211141 (2021).

[16] C. Moreau and K. Ishimoto, Driving a microswimmer with wall-induced flow, Micromachines 12, 1025 (2021).

[17] J. Słomka, U. Alcolombri, E. Secchi, R. Stocker, and V. I. Fernandez, Encounter rates between bacteria and small sinking particles, New J. Phys. 22, 043016 (2020).

[18] D. Saintillan and M. J. Shelley, Instabilities and Pattern Formation in Active Particle Suspensions: Kinetic Theory and Continuum Simulations, Phys. Rev. Lett. 100, 178103 (2008).

[19] M. -C. Kim and C. Klapperich, A new method for simulating the motion of individual ellipsoidal bacteria in microfluidic devices, Lab Chip 10, 2464 (2010).

[20] Note that we distinguish between the swimmer frame and the swimmer basis, with the latter simply being the orthonormal triad $\left\{\boldsymbol{e}_{x}, \boldsymbol{e}_{y}, \boldsymbol{e}_{z}\right\}$, which can form a basis of both the laboratory and the swimmer-fixed frames.

[21] C. Pozrikidis, Boundary Integral and Singularity Methods for Linearized Viscous Flow (Cambridge University Press, Cambridge, 1992).

[22] In writing this, we are minimally abusing notation by interpreting $\tilde{\boldsymbol{x}}^{S}$ as a two-dimensional vector field in order to write the scalar product between it and $\hat{\mathcal{L}} \boldsymbol{U}^{r}$, which we will continue to do throughout, noting that this is unambiguous as $\tilde{\boldsymbol{x}}^{S} \cdot \boldsymbol{e}_{z} \equiv 0$.

[23] If $\boldsymbol{A}$ were not invertible, then there would exist a nontrivial force- and torque-free motion when $\gamma=0$ and $\omega=0$, violating conservation of energy.

[24] C. M. Bender and S. A. Orszag, Advanced Mathematical Methods for Scientists and Engineers I (Springer New York, 1999).

[25] J. Gray and G. J. Hancock, The propulsion of sea-urchin spermatozoa, J. Exp. Biol. 32, 802 (1955).

[26] See Supplemental Material at http://link.aps.org/supplemental/10.1103/PhysRevFluids.7.L022101 for a constructive example and an analysis of small angular drift.

[27] B. M. Friedrich, I. H. Riedel-Kruse, J. Howard, and F. Julicher, High-precision tracking of sperm swimming fine structure provides strong test of resistive force theory, J. Exp. Biol. 213, 1226 (2010).

[28] D. M. Woolley, Motility of spermatozoa at surfaces, Reproduction 126, 259 (2003). 